\title{
Consistency in compensatory eating responses following acute exercise in inactive, overweight and obese women
}

\author{
Jessica L. Unick ${ }^{1 *}$, Kevin C. O'Leary ${ }^{1}$, Leah Dorfman ${ }^{1}$, J. Graham Thomas ${ }^{1}$, Kelley Strohacker ${ }^{2}$ \\ and Rena R. Wing ${ }^{1}$ \\ ${ }^{1}$ Weight Control and Diabetes Research Center, The Miriam Hospital and Brown Medical School, 196 Richmond Street, \\ Providence, RI 02903, USA \\ ${ }^{2}$ University of Tennessee, Knoxville, TN, USA
}

(Submitted 26 August 2014 - Final revision received 25 November 2014 - Accepted 21 January 2015 - First published online 17 March 2015)

\section{Abstract}

It is often assumed that some individuals reliably increase energy intake (EI) post-exercise ('compensators') and some do not ('noncompensators'), leading researchers to examine the characteristics that distinguish these two groups. However, it is unclear whether EI post-exercise is stable over time. The present study examined whether compensatory eating responses to a single exercise bout are consistent within individuals across three pairs of trials. Physically inactive, overweight/obese women $\left(n 28, \mathrm{BMI} 30 \cdot 3(\mathrm{sD} 2 \cdot 9) \mathrm{kg} / \mathrm{m}^{2}\right)$ participated in three pairs of testing sessions, with each pair consisting of an exercise (30 min of moderate-intensity walking) and resting testing day. EI was measured using a buffet meal $1 \mathrm{~h}$ post-exercise/rest. For each pair, the difference in $\mathrm{EI}\left(\mathrm{EI}_{\mathrm{diff}}=\mathrm{EI}_{\mathrm{ex}}-\mathrm{EI}_{\mathrm{rest}}\right)$ was calculated, where $\mathrm{EI}_{\mathrm{ex}}$ is the $\mathrm{EI}$ of the exercise session and $\mathrm{EI}_{\text {rest }}$ is the $\mathrm{EI}$ of the resting session, and women were classified as a 'compensator' $\left(\mathrm{EI}_{\mathrm{ex}}>\mathrm{EI}_{\mathrm{rest}}\right.$ ) or 'non-compensator' $\left(\mathrm{EI}_{\mathrm{ex}} \leq \mathrm{EI}_{\mathrm{rest}}\right)$. The average EI on exercise days (3328.0 (sD 1686.2) kJ) was similar to those on resting days $(3269.4(\mathrm{SD} 1582 \cdot 4) \mathrm{kJ})(P=0.67)$. Although EI was reliable within individuals across the three resting days (intraclass correlation coefficient (ICC) $0.75,95 \%$ CI $0.60,0.87 ; P<0 \cdot 001$ ) and three exercise days (ICC $0.83,95 \%$ CI $0.70,0.91 ; P<0.001$ ), the ICC for $\mathrm{EI}_{\text {diff }}$ across the three pairs of trials was low (ICC $0 \cdot 20,95 \% \mathrm{CI}-0 \cdot 02,0 \cdot 45 ; P=0 \cdot 04$ ), suggesting that compensatory eating post-exercise is not a stable construct. Moreover, the classification of 'compensators'/'non-compensators' was not reliable $(\kappa=-0 \cdot 048 ; P=0 \cdot 66)$. The results were unaltered when 'relative' EI was used, which considers the energy expenditure of the exercise/resting sessions. Acute compensatory EI following an exercise bout is not reliable in overweight women. Seeking to understand what distinguishes 'compensators' from 'non-compensators' based on a single eating episode post-exercise is not justified.

\section{Key words: Exercise: Physical activity: Energy expenditure: Compensation: Obesity}

Previous studies have demonstrated that the variability in weight loss produced by exercise training programmes is large, such that some individuals lose weight while others maintain or even gain weight following months of supervised exercise $^{(1-4)}$. This heterogeneity in weight loss suggests that some individuals may be compensating or increasing energy intake (EI) in response to exercise. Engagement in compensatory eating behaviours in response to exercise could undermine the beneficial effect of exercise on energy balance, and possibly lead to weight gain over time. A greater understanding of why compensation occurs could have important clinical implications for weight control.

One approach to understanding compensatory eating post-exercise is to examine it acutely, within a laboratory setting. Similar to exercise training trials, findings from acute laboratory-based studies reveal a large degree of variability in compensatory eating post-exercise with approximately half of participants increasing EI ('compensators') and the other half not altering or decreasing EI post-exercise ('non-compensators'), when compared with a resting, control condition $^{(5-7)}$. This has led researchers to begin to try to distinguish 'compensators' from 'non-compensators', examining whether these two groups differ in their physiological or affective responses to exercise ${ }^{(7-10)}$.

However, a significant concern with this research is that we have yet to establish whether the acute compensatory eating response to exercise is consistent over time. That is, before we begin to examine behavioural and physiological characteristics of compensators and non-compensators using a laboratory paradigm, we must first determine whether the difference

Abbreviations: EE, energy expenditure; EI, energy intake; $\mathrm{EI}_{\text {diff }}$, difference in energy intake; $\mathrm{EI}_{\mathrm{ex}}$, energy intake of the exercise session; EI $\mathrm{rest}_{\text {, }}$ energy intake of the resting session; HR, heart rate; ICC, intraclass correlation coefficient; REI, relative energy intake; RPE, rating of perceived exertion. 
between an individual's EI post-exercise $v$. EI post-rest is similar across occasions. If compensatory eating is not reliable, it would suggest that trying to identify variables that distinguish compensators from non-compensators based on this laboratory paradigm may not be appropriate.

Prior studies have examined the consistency in EI in a resting condition following the administration of a dietary preload. These studies have revealed that EI is highly reliable when measured on multiple resting occasions in healthy males (intraclass correlation coefficient (ICC) $0 \cdot 89-0 \cdot 97)^{(11,12)}$ and overweight/obese males (correlation coefficient being 0.76 or 0.90 when an outlier was excluded $)^{(13)}$, indicating that in controlled settings there is little fluctuation in EI on a daily basis in these populations. Moreover, Laan et al. ${ }^{(14)}$ reported that EI $35 \mathrm{~min}$ post-exercise is highly reproducible (ICC 0.90) when measured on two separate days among physically active men and women with a BMI between 18 and $29 \mathrm{~kg} / \mathrm{m}^{2}$. However, with the exception of one smaller study ( $n$ 14) by Brown et al. ${ }^{(15)}$, the consistency in "compensation' (e.g. EI measured on the exercise day minus EI measured on the resting day) has not been examined. Furthermore, the majority of studies that have examined the effect of exercise on appetite control have utilised trained, normal-weight males. However, research has suggested that trained individuals may be better able to regulate their energy needs, compared with those who are untrained, possibly due to deficient homeostatic feedback control of hunger and satiety in sedentary individuals ${ }^{(16-19)}$. In addition, women and overweight individuals may be more likely to compensate in response to exercise compared with men and those who are normal weight ${ }^{(20,21)}$. For example, there may be differences in appetite, cognitive factors, motivation to eat, eating behaviour characteristics such as dietary restraint or disinhibition, and gut peptides (e.g. ghrelin) between overweight/obese and normal-weight individuals ${ }^{(18,19,22,23)}$. While it still remains unclear how training status, sex or BMI influence EI following exercise, it is plausible to hypothesise that energy compensation in response to exercise is most likely to occur in untrained, overweight/obese women.

The purpose of the present study was to examine whether the difference in EI following a period of exercise and a period of rest is reliable across three separate pairs of exercise/resting trials in physically inactive, overweight/ obese women. A secondary aim was to determine whether classification of an individual as a 'compensator' $v$. 'noncompensator' is consistent over time. We specifically focused our investigation on women, given their large variability in EI post-exercise ${ }^{(5,6)}$. We also utilised a physically inactive sample, given that the majority of overweight/obese individuals do not exercise regularly. Furthermore, exercise is a recommended weight-loss strategy for overweight/obese individuals, and thus this research question may be the most clinically relevant in this population.

\section{Methods}

\section{Subjects}

Subjects were overweight and obese women (BMI $25-<35 \mathrm{~kg} / \mathrm{m}^{2}$ ) between the age of 18 and 45 years. All reported being physically inactive $(<60 \mathrm{~min} /$ week of moderate-intensity exercise), weight-stable $( \pm 10 \mathrm{lb}(4 \mathrm{~kg})$ over the past 6 months), relatively healthy (e.g. free of heart disease and diabetes, not taking any medications that would alter heart rate (HR) or metabolism, and no reported orthopaedic conditions that would have an impact on exercise), and sleeping an average of $>6 \mathrm{~h} /$ night. Subjects ate breakfast regularly and reported liking and being willing to eat at least $75 \%$ of the foods that were provided as part of the buffet meal, which was used to measure EI during the experimental testing sessions. The present study was conducted according to the guidelines laid down in the Declaration of Helsinki, and all procedures involving human subjects/patients were approved by the Miriam Hospital's Institutional Review Board. Written informed consent was obtained from all subjects/patients. The present study was registered at the ClinicalTrials.gov registry (study identifier: NCT01330329).

\section{Study protocol}

After undergoing an initial assessment visit, in which subjects underwent initial anthropometric testing and were oriented to study procedures, subjects participated in three pairs of testing visits, with each pair consisting of an exercise and resting testing day (totally six visits), using a randomised, counterbalanced design. Thus, the order of the testing visits differed for each participant; however, all participants had two pairs of testing visits in which the order was identical and one pair in which the order was reversed (e.g. pair 1: exercise first; pair 2: exercise first; pair 3: rest first). Visits within a pair were separated by $48-96 \mathrm{~h}$, while pairs of testing sessions were separated by at least $7 \mathrm{~d}$ (Fig. 1). All testing visits lasted approximately $3 \mathrm{~h}$, were performed at the same time of day

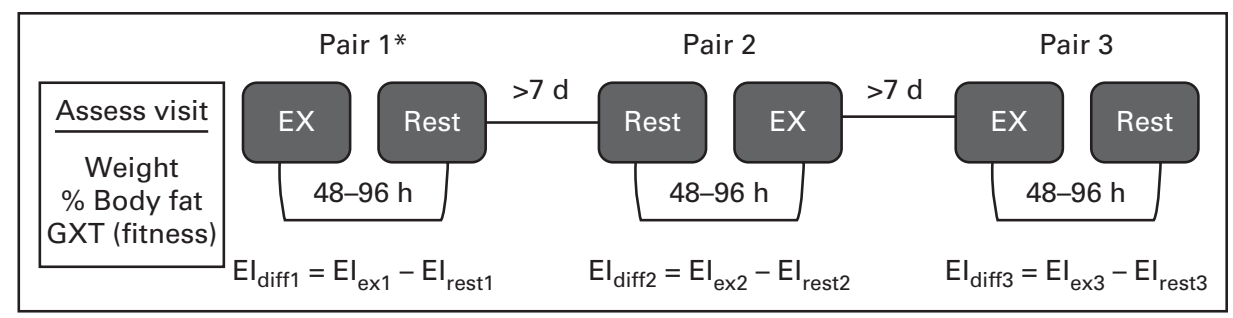

Fig. 1. Overview of the study. * Participants were randomised to a specific order of testing visits using a randomised, counter-balanced design. GXT, graded exercise test; $\mathrm{El}_{\text {diff, }}$ energy intake difference; $\mathrm{El}_{\mathrm{ex}}$, energy intake of the exercise session; $\mathrm{El}_{\text {rest }}$, energy intake of the resting session. 
( $\pm 30 \mathrm{~min}$ ), and were conducted in the morning (starting between 07.30 and 09.30 hours). Both exercise and resting testing days were identical, with the exception of a $30 \mathrm{~min}$, moderate-intensity treadmill bout on the exercise day and a $30 \mathrm{~min}$ period of seated rest on the resting day. Before each testing session, participants were instructed to (1) not consume any food or energy beverages past midnight, (2) refrain from exercising $24 \mathrm{~h}$ before their visit, (3) abstain from any caffeine or alcohol use $12 \mathrm{~h}$ before their visit, and (4) maintain regular sleeping habits. Research staff queried participants at the beginning of each testing visit to confirm compliance. Participants were asked to report the last time that they ate, exercised, or had caffeine or alcohol, and how many hours of sleep they had the previous night. If participants were non-compliant to the pre-testing recommendations, their testing visit was rescheduled. Following the completion of all six testing visits, participants completed the Three-Factor Eating Questionnaire ${ }^{(24)}$ that was used to assess dietary restraint and disinhibition.

\section{Assessment visit}

Height and weight were measured using standard procedures, and body composition was assessed using bioelectrical impedance (RJL Systems). Subjects completed a submaximal graded exercise test at $75 \%$ of age-predicted maximal HR. This graded exercise test allowed participants to become familiar with walking on a treadmill, provided a surrogate measure of fitness, and assisted in determining the starting treadmill grade for the initial exercise testing session (see below)

\section{Experimental testing session}

Fig. 2 provides an overview of the experimental testing sessions. Upon arrival, participants were informed of whether it would be an exercise or resting day, as not to bias them before the testing visit. Body weight was measured to ensure that weight did not change over time, and participants consumed a standardised meal replacement bar $(878.6 \mathrm{~kJ}$ (210 kcal), $47 \%$ carbohydrate, $26 \%$ fat and $27 \%$ protein). Participants then completed several computer tasks and questionnaires, used to blind participants to the true purpose of the study (i.e. measurement of EI). At $45 \mathrm{~min}$ after arrival, participants either rested quietly or exercised for $30 \mathrm{~min}$ while watching a standardised video (from the British Broadcasting Corporation's Planet Earth video series). Immediately following this exercise or resting session, participants again completed the same series of questionnaires and computer tasks. Following these tasks, participants sat quietly by themselves and were given the option to read or to continue watching the video until the start of the feeding session. At $1 \mathrm{~h}$ following the cessation of the exercise or seated rest, participants were provided ad libitum access to a buffet meal (see additional details below). The questionnaires and computer tasks were repeated following the feeding session.

\section{Exercise session}

During the first exercise visit, subjects walked on a treadmill (Spirit XT685; Spirit Fitness) at $3.0 \mathrm{mph}$ at a grade that elicited a HR between 70 and $75 \%$ of age-predicted maximal HR for $30 \mathrm{~min}$. HR was recorded every minute using the T31 HR monitor (Polar), and the grade of the treadmill was adjusted appropriately if the subject's HR fell outside the target HR range for two consecutive minutes. If the subject's HR was above the target $\mathrm{HR}$ range at a $0 \%$ treadmill grade, the speed of the treadmill was reduced. Any adjustments made to the grade or speed of the treadmill were noted so that an identical exercise protocol could be employed during the second and third exercise testing visits (i.e. changes in speed/grade from visit 1 were duplicated in visits 2 and 3, regardless of whether the participant's HR fell out of the targeted HR range). The energy expenditure (EE) of the exercise session was calculated using the American College of Sports Medicine's prediction equations for the EE of walking ${ }^{(25)}$. Ratings of perceived exertion (RPE) were assessed every $5 \mathrm{~min}$ during exercise using Borg's 6-20 RPE scale ${ }^{(26)}$. The EE of the resting session was calculated using the American College of Sports Medicine's EE prediction equation, assuming a resting value of $3.5 \mathrm{ml} / \mathrm{kg}$ per $\mathrm{min}$.

\section{Measurement of energy intake and macronutrient composition}

Subjects were provided with ad libitum access to a buffet-style meal starting $1 \mathrm{~h}$ post-exercise/rest, and efforts were taken to blind subjects to the measurement of EI. Subjects ate alone, without any music or videos, and were given half an hour to consume as much food as desired. EI was assessed by weighing all foods before and following the feeding session while using the manufacturer's energy values and food tables to calculate total EI. The test meal consisted of bagels, cream cheese, jelly, three varieties of cereal, granola, yogurt, $1 \%$ milk, doughnut and canned fruit, all of which were provided in excess of expected consumption (Table 1).

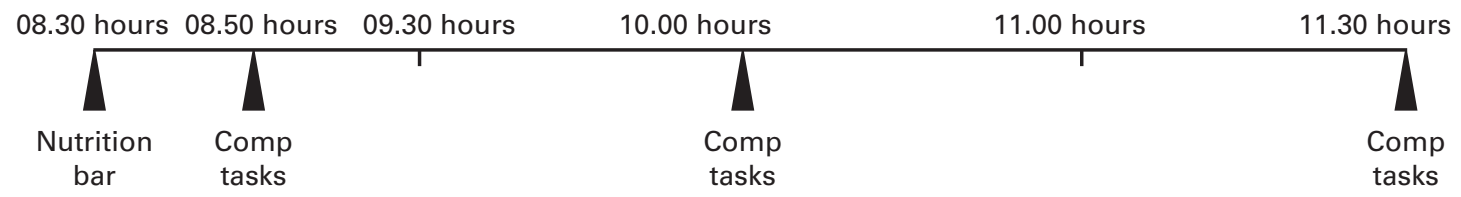

Fig. 2. Summary of the experimental testing visits. Comp tasks, completion of computer tasks and questionnaires. 
Table 1. Description of foods provided during the buffet meal

\begin{tabular}{|c|c|c|c|c|c|}
\hline Foods & Energy density (kcal/g) & Energy density $(\mathrm{kJ} / \mathrm{g})$ & Amount provided $(\mathrm{g})$ & Total energy (kcal) & Total energy (kJ) \\
\hline Plain bagel & $2 \cdot 79$ & 11.6 & 133.9 & 373.7 & 1563.5 \\
\hline Cinnamon raisin bagel & $2 \cdot 79$ & 11.6 & $135 \cdot 8$ & $379 \cdot 1$ & $1586 \cdot 2$ \\
\hline Plain cream cheese & 3.33 & 13.9 & $184 \cdot 2$ & 614.0 & 2568.9 \\
\hline Strawberry cream cheese & 3.33 & 13.9 & 206.4 & 688.1 & $2879 \cdot 0$ \\
\hline Strawberry preserves & $2 \cdot 50$ & $10 \cdot 5$ & 340.0 & $850 \cdot 0$ & $3556 \cdot 4$ \\
\hline Chocolate donettes & $4 \cdot 79$ & $20 \cdot 0$ & $129 \cdot 0$ & $618 \cdot 2$ & $2586 \cdot 5$ \\
\hline Powdered donettes & 4.39 & 18.4 & $127 \cdot 4$ & 558.6 & $2337 \cdot 2$ \\
\hline Vanilla yogurt & 0.84 & 3.5 & $825 \cdot 6$ & $691 \cdot 0$ & $2891 \cdot 1$ \\
\hline Light strawberry yogurt & 0.57 & $2 \cdot 4$ & $832 \cdot 2$ & $476 \cdot 6$ & $1994 \cdot 1$ \\
\hline Fruit cocktail & 0.48 & 2.0 & $415 \cdot 0$ & $200 \cdot 8$ & $840 \cdot 1$ \\
\hline Granola & 4.39 & $18 \cdot 4$ & $680 \cdot 4$ & $2984 \cdot 1$ & 12485.5 \\
\hline Cheerios & 3.57 & 14.9 & $256 \cdot 2$ & 914.9 & 3827.9 \\
\hline Golden Grahams & 3.87 & $16 \cdot 2$ & $792 \cdot 2$ & 3066.5 & $12830 \cdot 2$ \\
\hline Rice Krispies & 3.94 & $16 \cdot 5$ & 299.7 & $1180 \cdot 6$ & $4939 \cdot 6$ \\
\hline $1 \%$ Milk & 0.46 & 1.9 & $1085 \cdot 8$ & $497 \cdot 7$ & 2082.4 \\
\hline
\end{tabular}

\section{Statistical analysis}

The difference in $\mathrm{EI}\left(\mathrm{EI}_{\mathrm{diff}}\right)$ between sessions was calculated as the EI of the exercise session $\left(\mathrm{EI}_{\mathrm{ex}}\right)$ minus the EI of the resting session $\left(\mathrm{EI}_{\text {rest }}\right)$, and was calculated for each of the three pairs of exercise/resting trials $\left(\mathrm{EI}_{\mathrm{diff}}=\mathrm{EI}_{\mathrm{ex}}-\mathrm{EI}_{\text {rest }}\right)$. Relative energy intake (REI) was also calculated for each testing visit by subtracting the EE of the exercise or resting session from the EI on that testing day $(\mathrm{REI}=\mathrm{EI}-\mathrm{EE})$. The difference in $\mathrm{REI}\left(\mathrm{REI}_{\text {diff }}\right)$ was calculated in a similar manner $\left(\mathrm{REI}_{\mathrm{diff}}=\mathrm{REI}_{\mathrm{ex}}-\mathrm{REI}_{\text {rest }}\right)$. ICC were calculated to examine whether $\mathrm{EI}_{\mathrm{ex}}, \mathrm{EI}_{\text {rest }}, \mathrm{EI}_{\text {diff }}$ and $\mathrm{REI}_{\text {diff }}$ were similar across the three pairs of trials. The higher the ICC value (range $0-1 \cdot 0$ ), the greater the consistency in the measure, with ICC values $<0.40$ indicating 'poor' agreement, those between 0.40 and 0.59 indicating 'fair' agreement, those between 0.60 and 0.74 indicating 'good' agreement, and those between 0.75 and 1.00 indicating 'excellent' agreement $^{(27)}$. A $3 \times 2$ (time $\times$ condition) repeated-measures ANOVA was used to examine the change in EI over time on exercise, relative to resting testing days.

The classification of an individual as a 'compensator' or 'non-compensator' was performed using both absolute EI and REI for each of the three pairs of trials. If $\mathrm{EI}_{\mathrm{ex}}>\mathrm{EI}_{\text {rest }}$, an individual was classified as a 'compensator', and if $\mathrm{EI}_{\mathrm{ex}}<$ $\mathrm{EI}_{\text {rest }}$, they were classified as a 'non-compensator', when absolute EI scores were utilised. For REI, an individual was considered to be a 'compensator' if EI post-exercise exceeded the sum of their resting EI plus the net EE of the exercise bout (i.e. $\mathrm{REI}_{\mathrm{ex}}>\mathrm{REI}_{\mathrm{rest}}$ ). A 'non-compensator' was an individual whose EI post-exercise did not exceed the sum of the resting EI plus the net $\mathrm{EE}$ of the exercise bout (i.e. $\mathrm{REI}_{\mathrm{ex}}<\mathrm{REI}_{\text {rest }}$ ). A modified $\kappa$ coefficient ${ }^{(28)}$ was calculated to indicate the degree to which individuals tend to fall into the same category ('compensator' $v$. 'non-compensator') across the three pairs of trials. A statistically significant positive $\kappa$ value would indicate that the categorisation of a 'compensator' or 'non-compensator' was reliable within persons across the three pairs of trials.

Paired samples $t$ tests were used to examine whether there was a difference in EI or REI on resting days compared with EI or REI on exercise days within each pair. Statistical analyses were performed using SPSS version 18.0 for Windows
(SPSS, Inc.). All data are reported as means and standard deviations. Statistical significance was set at $P<0 \cdot 05$.

\section{Results}

\section{Subjects}

A total of thirty-four subjects participated in the present study. Of these, twenty-eight completed all six experimental testing visits and thus were included in the analyses. On average, participants were 33.1 (SD 9.6) years of age, had a BMI of $30 \cdot 3$ (SD 2.9$) \mathrm{kg} / \mathrm{m}^{2}$, had a body fat percentage of 37.9 (SD 5.4$) \%$, and $61 \%$ were Caucasian. Dietary restraint and disinhibition scores were 7.9 (SD 3.7) and 9.3 (SD 3.2), respectively. The estimated metabolic equivalent (MET) value at $75 \%$ of agepredicted maximal HR was 5.2 (SD 0.9) MET. Subjects' weight did not change over the six experimental visits $(P=0 \cdot 10)$.

\section{Exercise and resting sessions}

Each subject completed three identical exercise bouts in which the average speed and grade of the treadmill were $2 \cdot 92$ (SD 0.14) mph and 2.16 (SD 1.98)\%, respectively. Averaged across the three exercise sessions (EX), subjects exercised at $70 \cdot 8$ (SD 3.0)\% $\mathrm{HR}_{\max }$; however, $\mathrm{HR}$ was lower during $\mathrm{EX}_{2}$ (70.0 (SD 3.6)\% HR $\mathrm{HR}_{\max }$ ) compared with $\mathrm{EX}_{1}(72 \cdot 2$ (SD 1.8)\% $\left.\mathrm{HR}_{\max } ; \quad P=0.001\right)$, with no differences in $\mathrm{HR}$ observed between the other exercise sessions $(P>0.05)$. The mean RPE throughout the $30 \mathrm{~min}$ exercise period was 11.5 (SD 2.0), with the RPE during $\operatorname{EX}_{1}(12 \cdot 0$ (SD 2.0)) being higher than that during $\mathrm{EX}_{2}\left(11 \cdot 3\right.$ (SD 2.0)) or $\mathrm{EX}_{3}$ (11.3 (SD 2.3); $P<0.05)$. The EE of each exercise bout was estimated to be 722.2 (SD 166.5) kJ (172.6 (sD 39.8) kcal), which was significantly greater than the estimated $\mathrm{EE}$ of the resting session $(179.5$ (sD $27 \cdot 2) \mathrm{kJ}(42.9(\mathrm{sD} 6.5) \mathrm{kcal} ; P<0.001))$.

\section{Energy intake}

Table 2 presents the absolute and REI and $\mathrm{EI}_{\mathrm{diff}}$ for each pair of trials. EI was similar over time $(P=0 \cdot 91)$, indicating that 
Table 2. Mean energy intake during the exercise and resting testing days for each pair of trials (Mean values and standard deviations)

\begin{tabular}{|c|c|c|c|c|c|c|c|c|c|c|c|c|}
\hline & \multicolumn{4}{|c|}{ Exercise session } & \multicolumn{4}{|c|}{ Resting session } & \multicolumn{4}{|c|}{$\mathrm{El}$ difference $\left(E \mathrm{I}_{\text {diff }}=\mathrm{El}_{\mathrm{ex}}-\mathrm{EI}_{\text {rest }}\right)$} \\
\hline & \multicolumn{2}{|c|}{ Mean } & \multicolumn{2}{|c|}{ SD } & \multicolumn{2}{|c|}{ Mean } & \multicolumn{2}{|c|}{ SD } & \multicolumn{2}{|c|}{ Mean } & \multicolumn{2}{|c|}{ SD } \\
\hline & $\mathrm{kJ}$ & kcal & $\mathrm{kJ}$ & kcal & $\mathrm{kJ}$ & kcal & $\mathrm{kJ}$ & kcal & $\mathrm{kJ}$ & kcal & $\mathrm{kJ}$ & kcal \\
\hline \multicolumn{13}{|c|}{ Absolute energy intake } \\
\hline Pair 1 & $3427 \cdot 1$ & $819 \cdot 1$ & $1823 \cdot 8$ & $435 \cdot 9$ & $3324 \cdot 2$ & 794.5 & $1946 \cdot 4$ & $465 \cdot 2$ & $102 \cdot 5$ & 24.5 & $998 \cdot 7$ & $238 \cdot 7$ \\
\hline Pair 2 & $3148 \cdot 9$ & $752 \cdot 6$ & $1810 \cdot 0$ & $432 \cdot 6$ & $3306 \cdot 2$ & $790 \cdot 2$ & $1738 \cdot 9$ & $415 \cdot 6$ & $-157 \cdot 3$ & $-37 \cdot 6$ & $1126 \cdot 3$ & $269 \cdot 2$ \\
\hline Pair 3 & $3408 \cdot 3$ & $814 \cdot 6$ & $1752 \cdot 3$ & $418 \cdot 8$ & $3177 \cdot 3$ & $759 \cdot 4$ & $1487 \cdot 8$ & $355 \cdot 6$ & 231.4 & $55 \cdot 3$ & $900 \cdot 4$ & $215 \cdot 2$ \\
\hline \multicolumn{13}{|c|}{ Relative energy intake } \\
\hline Pair 1 & $2705 \cdot 0$ & $646 \cdot 5$ & $1768 \cdot 6$ & $422 \cdot 7$ & $3145 \cdot 1$ & $751 \cdot 7$ & $1941 \cdot 4$ & $464 \cdot 0$ & $-400 \cdot 2$ & $-105 \cdot 2$ & $981 \cdot 1$ & 234.5 \\
\hline Pair 2 & $2426 \cdot 7$ & $580 \cdot 0$ & $1775 \cdot 7$ & $424 \cdot 4$ & $3126 \cdot 7$ & $747 \cdot 3$ & $1734 \cdot 7$ & $414 \cdot 6$ & $-700 \cdot 0$ & $-167 \cdot 3$ & $1104 \cdot 2$ & 263.9 \\
\hline Pair 3 & $2686 \cdot 1$ & $642 \cdot 0$ & 1717.5 & $410 \cdot 5$ & $2997 \cdot 8$ & $716 \cdot 5$ & 1482.8 & 354.4 & $-311 \cdot 7$ & -74.5 & $900 \cdot 0$ & $215 \cdot 1$ \\
\hline
\end{tabular}

there was no effect of the repeated use of the same buffet meal on EI. Furthermore, absolute EI was not significantly different between the exercise and resting days within each pair of trials $(P>0 \cdot 19)$. Repeated-measures ANOVA revealed no significant main effect of trial (pair 1, pair 2 or pair 3; $P=0.71$ ), condition (exercise $v$. rest; $P=0.66$ ) or trial $\times$ condition interaction $(P=0 \cdot 27)$ for absolute EI. When the EE of the exercise and resting sessions was taken into consideration, the REI was lower on the exercise day compared with the resting day within each pair ( $P=0 \cdot 002-0 \cdot 08)$. Repeated-measures ANOVA also revealed that there was a significant main effect of condition such that the average REI across the three exercise days was significantly less than those across the three resting days $(P=0 \cdot 001)$; however, there was no significant main effect of trial $(P=0 \cdot 71)$ or trial $\times$ condition interaction $(P=0 \cdot 27)$.

Although there was no difference in EI between the exercise and resting days at the group level (i.e. $\mathrm{EI}_{\text {diff }}$ was small), there was a large degree of variability in $\mathrm{EI}_{\mathrm{diff}}$ at the individual level (Fig. 3). Consistent with previous reports, compensatory eating was observed in approximately half of all trials when defined using absolute EI to identify compensation. Moreover, compensation was observed in $27 \%$ of all trials, when the REI criterion was utilised.

\section{Consistency in eating responses}

EI across the three resting days was reliable (ICC 0.75, $95 \%$ CI $0.60,0.87)$. Similarly, EI measured $1 \mathrm{~h}$ following exercise was also reliable across the three exercise days (ICC 0.83 , 95\% CI 0.70, 0.91). However, the ICC for $\mathrm{EI}_{\text {diff, calculated as }}$ $\mathrm{EI}_{\mathrm{ex}}-\mathrm{EI}_{\text {rest }}$, was very low (ICC 0.20, 95\% CI -0.02, 0.45), indicating only a 'slight agreement' in $\mathrm{EI}_{\text {diff }}$ across the three exercise/resting trials ${ }^{(29)}$. This poor agreement in $\mathrm{EI}_{\text {diff }}$ within an individual across the three pairs of trials is shown in Fig. 4.

Classification of an individual as a 'compensator' or 'non-compensator' based on the absolute $\mathrm{EI}_{\text {diff }}$ for each of the three pairs revealed a similar lack of consistency $(\kappa=-0.048 ; P=0.66)$, meaning that if an individual was categorised as a 'compensator' during the first exercise/resting pair, she would not necessarily be classified as a 'compensator' in the remaining two exercise/resting pairs. Only six of the twenty-eight participants (21\%) were consistently classified as either a 'compensator' ( $n$ 4) or 'non-compensator' ( $n$ 2) in all the three pairs of trials. There was also a lack of consistency when REI was used to classify an individual as a 'compensator' or 'non-compensator' $(\kappa=0.102 ; \quad P=0.51)$. Although there were far fewer instances of 'compensation' using the REI criteria, still less than $50 \%$ of the participants were consistently classified as either a 'compensator' ( $n$ 1) or 'non-compensator' ( $n$ 12) across all the three pairs of trials.

\section{Discussion}

Laboratory paradigms, which utilise an exercise day and a resting day, have been used to identify 'compensation' following an acute exercise bout. The present study investigated whether the identification of an individual as a 'compensator' or 'noncompensator' during an acute laboratory paradigm is consistent across multiple time points, when measured in inactive, overweight/obese women. Findings reveal that compensatory eating post-exercise is not consistent within an individual over time. That is, if this methodology was used and identified an individual as a 'compensator' during a single pair of exercise/ resting trials, there is a high likelihood that the individual would not be classified as a 'compensator' if measured at a later time point. This suggests that the classification of a person as a 'compensator' and 'non-compensator' based on a single pair of exercise/resting trials does not identify a reliable phenotype in this particular population.

The present findings are in agreement with the only other study to date to examine the consistency in $\mathrm{EI}_{\text {diff }}$ between the exercise and resting days. In a small sample ( $n$ 14) of overweight and sedentary women, Brown et al. ${ }^{(15)}$ reported a slightly greater ICC value for $\mathrm{EI}_{\text {diff }}$ across two pairs of exercise/resting trials (ICC $0 \cdot 37 v \cdot 0 \cdot 20$ observed in the present study), indicating a lack of consistency in compensatory eating post-exercise. The present findings not only confirm those reported by Brown et al. ${ }^{(15)}$ using a larger cohort, more stringent methodology (three $v$. two pairs of exercise/resting trials), and a much lower and more typical exercise EE for physically inactive, overweight women $(720 \mathrm{~kJ}(172 \mathrm{kcal}) v$. $1648.5 \mathrm{~kJ}(394 \mathrm{kcal}))$, but also add to the literature by examining the reliability of the dichotomous classification of 

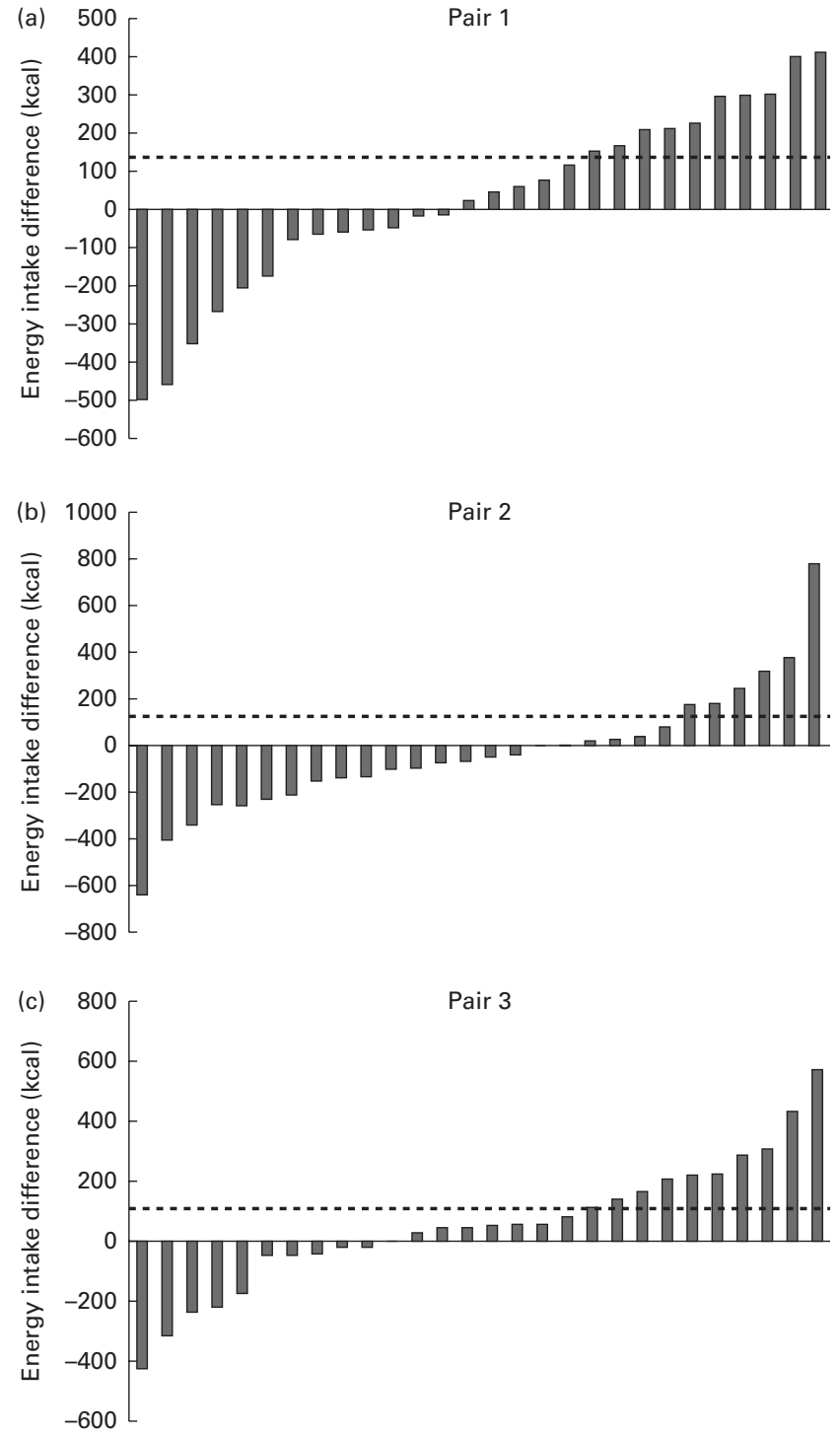

Fig. 3. Individual energy intake difference between the exercise $\left(E l_{e x}\right)$ and resting $\left(\mathrm{El}_{\text {rest }}\right)$ sessions for each pair of trials. Energy intake difference calculated as $\mathrm{El}_{\mathrm{ex}}-\mathrm{El}_{\text {rest }}$. Positive values indicate 'compensation' and negative values indicate 'non-compensation'. The dashed line reflects the net energy expenditure $\left(\mathrm{EE}_{\mathrm{ex}}-\mathrm{EE}_{\text {rest }}\right)$ of the exercise session; thus participants above the dashed line would be classified as 'compensators' using the relative energy intake criteria for compensation. To convert energy intake difference in kcal to $\mathrm{kJ}$, multiply by $4 \cdot 184$.

an individual as a 'compensator' or 'non-compensator' across trials. Together, these two studies demonstrate that compensatory eating in response to exercise is not reliable within inactive, overweight/obese individuals when measured across multiple time points. However, it could be argued that the population used within these studies has the poorest appetite regulation; thus, future studies are needed to examine whether there is also a lack of consistency observed in compensatory eating post-exercise within other populations that may have better appetite control (e.g. males, lean or physically active individuals).

Given that prior studies examining 'compensation' have utilised only a single pair of exercise/resting trials, we also compared our findings from a single pair of exercise/resting trials with previous reports. As shown in Fig. 3, there was a large degree of individual variability in $\mathrm{EI}_{\text {diff }}$ within any given pair of exercise/resting trials, with approximately an equal number of 'compensators' and 'non-compensators', as has been reported previously ${ }^{(5-7)}$. Moreover, as with previous studies in overweight/obese women, in the present study, there was no evidence of compensation at the group level (mean $\mathrm{EI}_{\text {diff }}$ ranging from $-154.8 \mathrm{~kJ}(-37 \mathrm{kcal})$ in pair 2 to $230 \cdot 1 \mathrm{~kJ}$ ( $55 \mathrm{kcal}$ ) in pair 3 ). Thus, although compensatory eating was not reliable within an individual over time, it appears that at any given measurement period, there will be individuals who eat more and others who eat less after exercise compared with rest, cancelling out one another at the group level. Future studies should begin to examine whether there are day-to-day variations in both psychological (e.g. mood, fatigue, hedonic or non-homeostatic factors) and physiological factors (e.g. HR response to exercise, fluctuations in hormones and hunger) that may contribute to compensatory eating within an individual on one occasion but not another.

From a clinical perspective, it is also important to consider the present findings and how they may relate to the role of exercise in weight control. Although it has never been tested, there is an underlying assumption that those who compensate by increasing EI acutely post-exercise in a laboratory setting are also the same individuals who lose less weight than expected (based on exercise-induced EE) when engaging in a longer-term exercise training programme, due to this compensatory eating mechanism. While the present study was not designed to examine this hypothesis, the lack of consistency observed suggests that other factors, besides acute compensatory eating, may probably explain why some individuals gain weight (or lose less weight than expected) while others lose significant amounts of weight with exercise training. As noted by Boutcher \& Dunn ${ }^{(30)}$, this variability in weight loss could be attributed to a variety of behavioural, physiological or inherited characteristics. Additional research is needed to identify the mechanisms explaining this variability in response.

Although the aim of the present study was to examine the consistency in $\mathrm{EI}_{\mathrm{diff}}$ across three exercise/resting trials, the study design also allowed for the examination of the consistency in EI following a $30 \mathrm{~min}$ rest period and following a $30 \mathrm{~min}$ exercise bout. Similar to previous reports, findings reveal that EI following a period of rest was reliable over time, suggesting that a buffet meal is a reliable method for assessing EI within a laboratory. However, the ICC value reported in the present study (ICC 0.75 ) was slightly lower than what has been reported previously following a period of rest in healthy, normal-weight men (ICC 0.86-0.97) ${ }^{(11,12,31)}$, and in trained males and females with a BMI between 18 and $29.9 \mathrm{~kg} / \mathrm{m}^{2}$ (ICC 0.86$)^{(14)}$. It is possible that the lower ICC value observed in the present study was due to the use of three testing days $(v .2 \mathrm{~d})$ or the fact that the present study used physically inactive, overweight/obese women, compared with males or trained individuals. Finally, the reliability of EI following $30 \mathrm{~min}$ of moderate-intensity treadmill walking in 


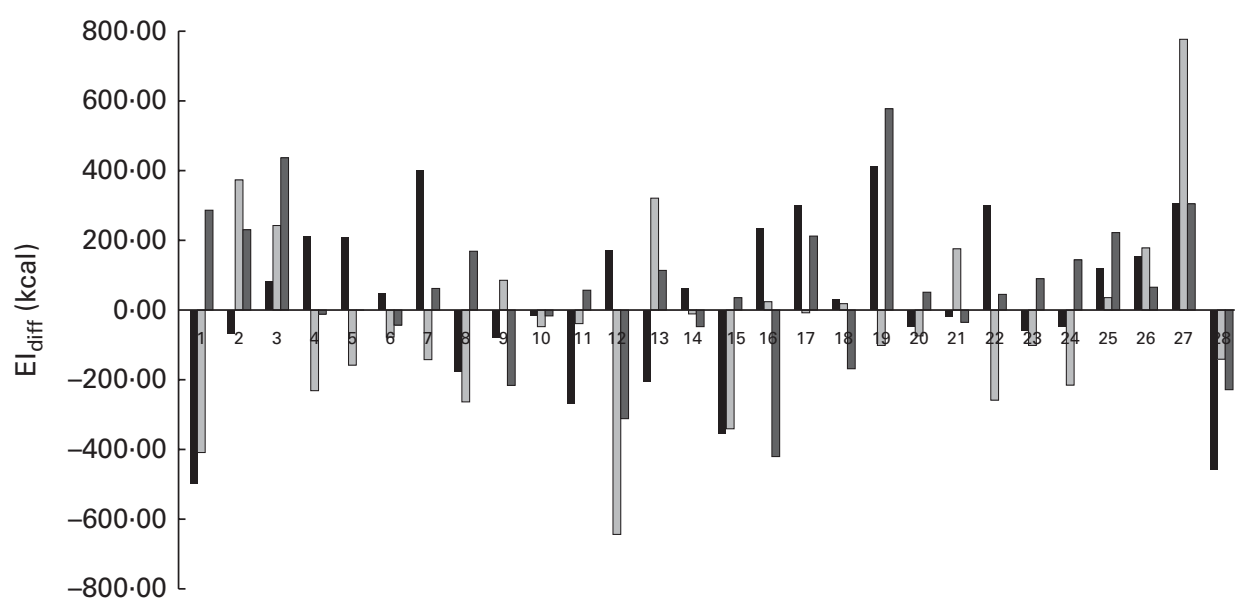

Fig. 4. Difference in energy intake $\left(E \mathrm{I}_{\text {diff }}\right)$ between the exercise $\left(E \mathrm{I}_{\mathrm{ex}}\right)$ and resting $\left(E \mathrm{I}_{\text {rest }}\right)$ sessions for each individual for each of the three pairs of trials. $E \mathrm{I}_{\text {diff }}$ calculated as $\mathrm{El}_{\mathrm{ex}}-\mathrm{El}_{\text {rest }}$, with a positive value indicating 'compensation' (i.e. an individual ate more after exercise compared with rest). $\mathbf{\square}, \mathrm{El}_{\text {diff1 }} ; \square, \mathrm{El}_{\text {diff2 }} ; \square, \mathrm{El}_{\text {diff3. }}$. To convert $\mathrm{El}_{\text {diff }}$ in kcal to $\mathrm{kJ}$, multiply by $4 \cdot 184$.

the present study was high (ICC 0.83 ) and very similar to that reported by Laan et al. ${ }^{(14)}$ (ICC $0 \cdot 86$ ) following a $35 \mathrm{~min}$ bout of pedalling on a cycle ergometer. This suggests that there is consistency in meal consumption following exercise.

The strength of the present study was the use of a rigorous methodology that utilised three pairs of identical exercise/ resting trials (as opposed to two pairs), a larger than typical sample size, a standardised breakfast administered in person, and a buffet-meal that consisted of a wide selection of food items $v$. a single dietary item. In addition, the present study utilised physically inactive, overweight/obese women, a population for which the examination of compensatory eating is clinically relevant. However, the present study is not without limitations. First, while efforts were taken to blind subjects to the measurement of EI, it is not possible to know whether the delivery of a buffet meal in an unnatural environment could have had an impact on eating behaviours. However, if this were the case, it is likely that this would have equally altered EI at all testing visits, thereby not affecting the consistency measure. Second, the timing of the EI measurement in relation to one's menstrual cycle may have influenced EI. However, given that resting and exercise days within a pair were conducted within $48-96 \mathrm{~h}$ of one another, it is likely that individuals were within the same phase of their menstrual cycle during the exercise and resting sessions within a pair, thus having little influence on the $\mathrm{EI}_{\mathrm{diff}}$ measure. Third, while efforts were taken to ensure that the grade and speed of the treadmill remained constant across the three exercise trials, this resulted in the fluctuation of $\mathrm{HR}$ and RPE across the exercise days. Some may argue that these physiological or perceptual responses to exercise could influence EI. Thus, we also examined the ICC for $\mathrm{EI}_{\text {diff }}$ using only pairs 2 and 3, given that HR and RPE were nearly identical between these pairs, and still found a lack of consistency in $\mathrm{EI}_{\text {diff }}$ when measured over time (ICC 0.27). This suggests that these differences in HR and RPE probably had little effect on our findings. Fourth, the EE of the exercise session in the present study was fairly small $(723.8 \mathrm{~kJ}(173 \mathrm{kcal}))$; thus, future studies are needed to examine whether a similar lack of consistency in
$\mathrm{EI}_{\text {diff }}$ is also observed when exercise-induced EE is greater. Finally, EI was measured $1 \mathrm{~h}$ post-exercise/rest; thus, it is not known whether individuals altered their eating habits after leaving the laboratory and whether this differed across the testing days. However, even if this were the case, the findings from the present study still suggest that this type of laboratory paradigm, which is used to assess compensatory eating, may not be a completely adequate method for distinguishing 'compensators' from 'non-compensators'.

In conclusion, our findings suggest that compensatory eating ( $\left.\mathrm{EI}_{\text {diff }}\right)$ in response to an exercise bout is not consistent when measured at multiple time points in physically inactive, overweight/obese women. Thus, using a laboratory paradigm with a single exercise and resting session to identify 'compensators' and 'non-compensators' and then seeking to identify other differences that distinguish these two groups may not be appropriate in this population. Future studies should examine how differences in participants' psychological or physiological responses on specific exercise and/or resting trials may contribute to differences in EI.

\section{Acknowledgements}

The present study was funded by a grant awarded to Dr Unick from the National Cancer Institute (1R03CA162965-01A1).

The authors' contributions are as follows: J. L. U., R. R. W., and J. G. T. contributed to the study design, J. L. U., K. C. O'. L., L. D., and K. S. assisted with data collection, J. L. U. and J. G. T. performed the data analyses, and J. L. U. wrote the manuscript. All authors contributed to the discussion and interpretation of the data, and reviewed and edited the manuscript.

There are no conflicts of interest.

\section{References}

1. Church TS, Martin CK, Thompson AM, et al. (2009) Changes in weight, waist circumference and compensatory responses with different doses of exercise among sedentary, overweight postmenopausal women. PLOS ONE 4, e4515. 
2. Donnelly JE \& Smith BK (2005) Is exercise effective for weight loss with ad libitum diet? Energy balance, compensation, and gender differences. Exerc Sport Sci Rev 33, 169-174.

3. Manthou E, Gill JM, Wright A, et al. (2010) Behavioral compensatory adjustments to exercise training in overweight women. Med Sci Sports Exerc 42, 1121-1128.

4. King NA, Hopkins M, Caudwell P, et al. (2008) Individual variability following 12 weeks of supervised exercise: identification and characterization of compensation for exercise-induced weight loss. Int J Obes (Lond) 32, 177-184.

5. Unick JL, Otto AD, Goodpaster BH, et al. (2010) Acute effect of walking on energy intake in overweight/obese women. Appetite 55, 413-419.

6. Finlayson G, Bryant E, Blundell JE, et al. (2009) Acute compensatory eating following exercise is associated with implicit hedonic wanting for food. Physiol Behav 97, 62-67.

7. Hopkins M, Blundell JE \& King NA (2014) Individual variability in compensatory eating following acute exercise in overweight and obese women. Br I Sports Med 48, 1472-1476.

8. Unick JL, Michael JC \& Jakicic JJ (2012) Affective responses to exercise in overweight women: initial insight and possible influence on energy intake. Psychol Sport Exerc 13, 528-532.

9. King NA, Caudwell P, Hopkins M, et al. (2007) Metabolic and behavioral compensatory responses to exercise interventions: barriers to weight loss. Obesity (Silver Spring) 15, 1373-1383.

10. Blundell JE, Stubbs JR, Hughes DA, et al. (2003) Cross talk between physical activity and appetite control: does physical activity stimulate appetite? Proc Nutr Soc 62, 651-661.

11. Arvaniti K, Richard D \& Tremblay A (2000) Reproducibility of energy and macronutrient intake and related substrate oxidation rates in a buffet-type meal. Br J Nutr 83, 489-495.

12. Nair NS, Brennan IM, Little TJ, et al. (2009) Reproducibility of energy intake, gastric emptying, blood glucose, plasma insulin and cholecystokinin responses in healthy young males. Br J Nutr 101, 1094-1102.

13. Horner KM, Byrne NM \& King NA (2014) Reproducibility of subjective appetite ratings and ad libitum test meal energy intake in overweight and obese males. Appetite 81, 116-122.

14. Laan DJ, Leidy HJ, Lim E, et al. (2010) Effects and reproducibility of aerobic and resistance exercise on appetite and energy intake in young, physically active adults. Appl Physiol Nutr Metab 35, 842-847.

15. Brown GL, Lean ME \& Hankey CR (2012) Reproducibility of 24-h post-exercise changes in energy intake in overweight and obese women using current methodology. Br I Nutr 108, 191-194.

16. Long SJ, Hart K \& Morgan LM (2002) The ability of habitual exercise to influence appetite and food intake in response to high- and low-energy preloads in man. $B r I$ Nutr 87, 517-523.

17. Martins C, Truby H \& Morgan LM (2007) Short-term appetite control in response to a 6-week exercise programme in sedentary volunteers. Br J Nutr 98, 834-842.

18. King NA, Tremblay A \& Blundell JE (1997) Effects of exercise on appetite control: implications for energy balance. Med Sci Sports Exerc 29, 1076-1089.

19. Martins C, Morgan L \& Truby H (2008) A review of the effects of exercise on appetite regulation: an obesity perspective. Int J Obes (Lond) 32, 1337-1347.

20. George VA \& Morganstein A (2003) Effect of moderate intensity exercise on acute energy intake in normal and overweight females. Appetite 40, 43-46.

21. Donnelly JE, Hill JO, Jacobsen DJ, et al. (2003) Effects of a 16-month randomized controlled exercise trial on body weight and composition in young, overweight men and women: the Midwest Exercise Trial. Arch Intern Med 163, 1343-1350.

22. Barkeling B, Rossner S \& Sjoberg A (1995) Methodological studies on single meal food intake characteristics in normal weight and obese men and women. Int J Obes Relat Metab Disord 19, 284-290.

23. Valera Mora ME, Scarfone A, Valenze V, et al. (2005) Ghrelin does not influence gastric emptying in obese subjects. Obes Res 13, 739-744.

24. Stunkard AJ \& Messick S (1985) The three-factor eating questionnaire to measure dietary restraint, disinhibition and hunger. J Psychosom Res 29, 71-83.

25. American College of Sports Medicine (2010) ACSM's Guidelines for Exercise Testing and Prescription, 8th ed. Philadelphia, PA: Wolters Kluwer/Lippincott Williams \& Wilkins.

26. Borg GV \& Linderholm H (1967) Perceived exertion and pulse rate during graded exercise in various age groups. Acta Medica Scandinavica 181, 194-206.

27. Cicchetti D (1994) Guidelines, criteria, and rules of thumb for evaluating normed and standardized assessment instruments in psychology. Psychol Assess 6, 284-290.

28. Fleiss JL (1971) Measuring nominal scale agreement among many raters. Psychol Bull 76, 378-382.

29. Landis JR \& Koch GG (1977) The measurement of observer agreement for categorical data. Biometrics 33, 159-174.

30. Boutcher SH \& Dunn SL (2009) Factors that may impede the weight loss response to exercise-based interventions. Obes Rev 10, 671-680.

31. Gregersen NT, Flint A, Bitz C, et al. (2008) Reproducibility and power of ad libitum energy intake assessed by repeated single meals. Am J Clin Nutr 87, 1277-1281. 